\title{
Painful chest wall swelling
}

\author{
D V Shah, S Ladhani, J M Joshi
}

A 70 year old man, a non-smoker, presented with a painful swelling over the right sternoclavicular joint and low grade continuous fever for a period of two months. The patient had been empirically started on antituberculous therapy on the suspicion of tuberculous osteomyelitis, but his fever and swelling persisted.

On clinical examination, a hard and tender swelling was present over the right sternoclavicular joint. The swelling was fixed to the underlying bone, but not to the overlying skin. The patient did not have any skin lesions. Examination of his respiratory system revealed no abnormality. Examination of other systems was unremarkable.

Blood investigations showed a haemoglobin concentration of $160 \mathrm{~g} / \mathrm{l}$. The total white blood cell count was $10.5 \times 10^{9} / 1$ with $72 \%$ polymorphonuclear cells, $26 \%$ lymphocytes, and $2 \%$ eosinophils. ELISA testing for HIV infection was negative. Other haematological and biochemical parameters were within normal limits.

Fine needle aspiration cytology of the swelling showed a few red blood cells, polymorphonuclear cells, lymphocytes, and a few epithelioid cells in a necrotic background. No granulomas or giant cells were seen.

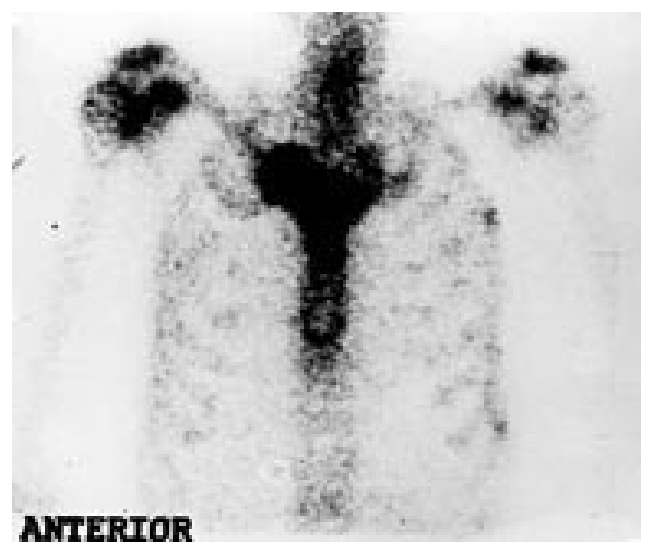

Figure 1 Bone scan.

Computed tomography (plain and contrast) of the thorax revealed a right sternoclavicular and first costoclavicular joints arthropathy with subchondral sclerosis and abnormal periarticular soft tissue mass. A technetium-99 bone scan was done (shown in fig 1) which revealed the diagnosis.

\section{Questions}

(1) What does the bone scan show?

(2) What is the diagnosis and name the associated syndrome. 
Answers

QUESTION 1

The bone scintigraphy shows an increased radiotracer uptake in the region of the right sternoclavicular joint and the manubrium sterni ("bullhead" sign). Increased uptake was also seen in the region of the left knee joint, the left ankle joint, D9 thoracic vertebrae, and the posterior end of the left eighth rib.

QUESTION 2

The diagnosis is sternocostoclavicular hyperostosis $(\mathrm{SCCH}) .{ }^{1}$ The associated syndromes are called SAPHO syndrome (an acronym for synovitis, acne, pustulosis, hyperostosis, and osteitis), ${ }^{12}$ CRMO (chronic recurrent multifocal osteomyelitis), ${ }^{12} \mathrm{ACW}$ syndrome (anterior chest wall), ${ }^{2}$ or PAO (pustulotic arthroosteitis). ${ }^{13}$

\section{Discussion}

SCCH is observed mainly in young and middle aged adults, and is rarely seen above 60 years of age. ${ }^{1}$ Bone and joint involvement are the commonest findings. Patients present with pain and swelling of gradual onset, most commonly involving the medial end of the clavicle and the manubrium sterni. Involvement of only one clavicle and the adjacent part of the manubrium sterni are also a frequent event. The ribs are similarly involved, with changes occurring in the anterior costochondral junction and/or in the posterior costal arch, leading to limitation of the thoracic cage mobility. ${ }^{1}$ The swelling might also present as a solitary neck mass or as thoracic sinus formation. ${ }^{45}$ Flat bones like the ileum, the mandible, long bones, or spine could also be involved in the disease process. ${ }^{1}$ The involved bone shows sclerotic changes with periosteal, articular, or periarticular inflammation. ${ }^{2}$

The skin involvement in SAPHO and PAO is usually in the form of palmoplantar pustulosis, palmoplantar pustular psoriasis, or severe acne known as acne conglomerata or acne fulminans. Though skin involvement is a common feature, the absence of skin lesions at the time of presentation is only apparent because the skin lesions may have been transitory, or may develop decades after the bony manifestation. The patient may have other manifestations like thoracic outlet syndrome, thrombosis of the subclavian vein, or superior vena cava syndrome. $^{1}$

Diagnosis can be made by radiography. Bone scintigraphy using radiotracer material like technetium-99 is the imaging modality of choice. It reveals hot spots in the areas of increased uptake. "Bullhead" sign refers to increased uptake by the medial end of the clavicles and manubrium sterni, corresponding to a bull's head. This sign is a typical and a highly specific manifestation of SSCH syndrome and helps to confer the diagnosis. ${ }^{1}$ Also a bone scan is able to detect early bone involvement, which would not yet be seen radiographically. Ultrasonography, computed tomography, and magnetic resonance imaging contribute little to the identification and location of the lesion. ${ }^{1}$
Learning points

- Sternocostoclavicular hyperostosis (SCCH) is a benign condition that involves bones and joints.

- $\mathrm{SCCH}$ is seen in the young and middle aged, rarely in old age.

- Associated syndromes include SAPHO, PAO with skin involvement.

- Diagnosis is by bone scan with classical "bullhead sign".

- Treatment consists of anti-inflammatory drugs, rarely in severe cases with steroids.

- SCCH should be considered in the differential diagnosis of bacterial osteomyelitis, Paget's disease, Ewing's sarcoma, and infectious spondylodiscitis.

There are no specific markers for these syndromes. Blood counts are usually normal. A moderate increase in $\mathrm{C}$ reactive proteins, erythrocyte sedimentation rate, and alpha-2 and gammaglobulins may be present. Histopathological examination of the biopsied tissue shows hyperostosis. Later in the course of the disease an infiltrate of mononuclear cells, lymphocytes, plasmocytes and a few multinucleated cells may be seen. ${ }^{1}$ Various theories have been postulated regarding the aetiopathogenesis. A reactive osteomyelitis is potentially triggered by saprophytes or caused by infectious agents like Propriobacterium acne, which has been found at the site of a lesion in many cases. The link with seronegative spondylarthritis also suggests a genetic predisposition, loosely associated with HLA-B27.

The treatment involves the use of antiinflammatory drugs. The duration of treatment has not been standardised. Corticosteroids may be used in the most severe form. Colchicine and sulphasalazine may prove to be successful. ${ }^{1}$ Thus the recognition of SCCH syndrome is essential to differentiate this benign condition from other serious involvements like bacterial osteomyelitis, infectious spondylodiscitis, Ewing's sarcoma, and Paget's disease. ${ }^{5}$ This can protect an individual from unnecessary therapeutic procedures and unnecessary medications. ${ }^{13}$

\section{Final diagnosis}

Sternocostoclavicular hyperostosis.

1 Kahn M F, Chamot A M. SAPHO syndrome. Rheum Dis Clin North Am 1992;18:225-43.

2 Schilling F, Kessler S. SAPHO syndrome: clinicorheumatologic and radiologic differentiation and classification of a patient sample of 86 cases. $Z$ Rheumatol 2000;59: $1-2$.

3 Freyschmidt J, Sternberg A. The bullhead sign: scintigraphic pattern of sternocostoclavicular hyperostosis and pustulotic arthroosteitis. European Radiology 1998;5:80781.

4 Finkelstein DM, Noyek AM. Sternocostoclavicular hyperostosis (SCCH) presenting as lung mass in the neck. $f$ Otolaryngol 1989;18:254-7.

5 Wilson GE, Evans CC. Sternocostoclavicular hyperostosis presenting with thoracic sinus formation. Thorax 1996;51: 